\title{
Viabilidade de uso da madeira de Cupressus torulosa para produção de painéis compensados
}

\author{
Viability of the use of wood of Cupressus torulosa for plywood production
Setsuo Iwakiri ${ }^{1}$, Rosilani Trianoski ${ }^{1}$, Helena Cristina Vieira ${ }^{1}$, Rafael Andrade ${ }^{1}$, Thaisa Mariana Santiago Rocha ${ }^{1}$ e Vitória Roberta Silva Ferreira ${ }^{1}$

\begin{abstract}
Resumo
Esta pesquisa teve como objetivo avaliar a qualidade de painéis compensados produzidos com lâminas de madeira de Cupressus torulosa em mistura com lâminas de Pinus taeda. Foram produzidos, em laboratório, compensados com cinco lâminas de 2,0 mm de espessura, sendo todas com lâminas de Cupressus e de Pinus, além da mistura destas nas capas e miolo do painel. A batida de cola foi formulada com $25 \%$ e $35 \%$ e gramatura de $360 \mathrm{~g} / \mathrm{m}^{2}$ (linha dupla). Os painéis foram prensados com temperatura de $140^{\circ} \mathrm{C}$, pressão específica de $10 \mathrm{kgf} / \mathrm{cm}^{2}$ e tempo de 10 minutos. A qualidade dos compensados foi avaliada por meio das seguintes propriedades: massa específica aparente, flexão estática e cisalhamento da linha de cola. $\mathrm{O}$ aumento no teor de sólidos da batida de cola não resultou em melhora das propriedades dos painéis, indicando a possibilidade de colagem de lâminas com menor consumo de adesivo. Os resultados dos ensaios indicaram que a espécie Cupressus torulosa apresenta grande potencial para produção de painéis compensados, puro, ou em mistura com Pinus taeda.
\end{abstract}

Palavras-chave: laminas de madeira, fenol-formaldeído, cisalhamento da linha de cola.

\begin{abstract}
The aim of this research was to evaluate the quality of plywood produced from wood veneers of Cupressus torulosa in mixture with veneer of Pinus taeda. In the laboratory plywood with five veneers of $2.0 \mathrm{~mm}$ thickness were produced, all with Cupressus and Pinus veneers, aside from the mixing of these species in the face and core of the panel. The glue beat was formulated with $25 \%$ and $35 \%$ of solid contents and amount of glue of $360 \mathrm{~g} / \mathrm{m}^{2}$ (double line). The panels were pressed with a temperature of $140^{\circ} \mathrm{C}$, specific pressure of $10 \mathrm{kgf} / \mathrm{cm}^{2}$ and a time of 10 minutes. The quality of the plywood was evaluated by means of the following properties: board apparent density, static bending and shear of the glue line. The increase in the solids content of the glue beat did not result in improvement of the properties of the panels, indicating the possibility of gluing the veneers with less adhesive consumption. The results of the tests indicated that Cupressus torulosa presents great potential for the production of plywood; either pure, or in mixture with Pinus taeda.
\end{abstract}

Keywords: wood veneer, phenol-formaldehyde, glue line shear test

\section{INTRODUÇÃO}

As indústrias de painéis compensados no Brasil utilizam, como matéria-prima, madeira de Pinus e de espécies tropicais da Amazônia. Tendo em vista a pressão ambiental sobre a utilização de madeiras tropicais e a necessidade de grandes áreas de plantios de Pinus para atender à demanda das indústrias de compensados e, também, de outros segmentos como papel e celulose, painéis particulados e de fibras, estudos sobre espécies alternativas de rápido crescimento têm sido amplamente realizados no Brasil, desde a década de 1990.

Além das espécies de Eucalyptus, vários estudos têm sido desenvolvidos para avaliar o comportamento de algumas espécies para produção de lâminas e compensados. Pinto e Iwakiri (2013) avaliaram o rendimento em laminação e qualidade dos painéis compensados de Cryptomeria japônica; Trianoski et al. (2015) estudaram o comportamento da madeira de Melia azedarach na produção de compensados fenólicos com diferentes gramaturas e tempos de prensagem; Bortoletto Junior e Belini

1. Departamento de Engenharia e Tecnologia Florestal, Universidade Federal do Paraná - UFPR. Curitiba / PR, Brasil. E-mail: setsuo.ufpr@gmail.com 
(2002) avaliaram a qualidade de compensado produzido com madeira de Schizolomibum parahyba; Iwakiri et al. (2013) avaliaram o comportamento da madeira de Sequoia sempervirens na qualidade de compensados fenólicos. Para todas as espécies citadas, os pesquisadores encontraram resultados que indicam a sua potencialidade na produção de lâminas e compensados.

O gênero Cupressus é classificado como uma conífera, sendo conhecido como ciprestes do Himalaia, com ocorrência natural no sudoeste da China, nordeste da Índia e Nepal. A sua madeira, extraída de alguns plantios em pequena escala, ou experimentais, são utilizadas no Quênia; como fonte de combustível na Guatemala; para produção de polpa na Venezuela (OKINO et al., 2005). Plantios experimentais no Brasil têm apresentado boas taxas de crescimento e, em função da sua madeira apresentar densidade média a baixa, apresenta grande potencial como matéria-prima para as indústrias de papel e celulose, laminados e compensados, além de painéis de partículas e de fibras (HASLET, 1986).

As limitações relacionadas à baixa densidade da madeira e menores diâmetros das árvores de espécies de florestas plantadas de rápido crescimento, podem ser minimizadas com uso de tecnologias de colagem para fabricação de produtos reconstituídos de madeira. Bendsten (1978) afirma que, para se alcançar êxito no uso de recursos florestais provenientes de florestas plantadas, os conceitos tradicionais devem ser modificados e adaptados às características da matéria-prima disponível.

Na produção de compensados, os fatores relacionados ao processo físico-químico envolvido na colagem de lâminas de madeira são de suma importância na qualidade do produto final (MARRA, 1992). Dentre os fatores mais importantes no processo produtivo de compensados podem se destacar: tipo de resina, para uso interno e externo; a formulação da batida de cola; a gramatura; e os parâmetros do ciclo de prensagem - temperatura, tempo e pressão. A formulação da batida de cola indica a proporção de resina na mistura e é definida em função da qualidade e custo de produção desejada (BALDWIN, 1995; SELLERS, 1993).

Outro fator importante é a densidade da madeira, tendo em vista as interações que ocorrem entre a porosidade da madeira e a absorção do adesivo na formação da ligação adesiva entre as lâminas. Marra (1992) afirma que, madeiras de baixa densidade absorvem maior quantidade de adesivo em função da maior porosidade; outros fatores como $\mathrm{pH}$ e extrativos presentes na madeira, são também importantes, podendo interferir na cura do adesivo durante o processo de prensagem. $\mathrm{O}$ autor afirma ainda que, o pH muito baixo de algumas espécies pode retardar a polimerização da resina fenol-formaldeído, que cura no meio alcalino, reduzindo a resistência da ligação adesiva. Já Baldwin (1995) afirma que, alguns extrativos presentes na madeira podem dificultar durante a prensagem a quente, o processo de movimentação de água na forma de vapor das linhas de cola para outra, e posterior liberação para o ambiente externo. Este processo, sendo muito lento, terá como consequência o aumento da pressão interna de vapor, resultando no "estouro" no momento da abertura da prensa e da delaminação do painel.

Perante o exposto, esta pesquisa foi desenvolvida com o objetivo de avaliar o potencial de uso de lâminas de madeira de Cupressus torulosa, em mistura com Pinus taeda, para produção de painéis compensados, utilizando resina fenol-formaldeído com diferentes teores de sólidos da batida de cola.

\section{MATERIAL E MÉTODOS}

Foram utilizadas, nesta pesquisa, lâminas de madeiras de Cupressus torulosa e Pinus taeda, com dimensões de 500,0 x 500,0 x 2,0 mm. As lâminas foram secas em estufa, até o teor de umidade médio de $8 \%$, e separadas em lotes de cinco lâminas para a composição do painel. As batidas de cola foram preparadas com teor de sólidos de 25\% e 35\%, sendo aplicadas sobre uma das faces da lâmina, com gramatura de $360 \mathrm{~g} / \mathrm{cm}^{2}$ (linha dupla). Os painéis foram compostos com todas as lâminas de Cupressus torulosa e de Pinus taeda, além da mistura destas espécies, sendo capas de Cupressus torulosa com miolo de Pinus taeda e capas de Pinus taeda com miolo de Cupressus torulosa.

Os painéis foram prensados à temperatura de $140^{\circ} \mathrm{C}$, pressão específica de $10 \mathrm{kgf} / \mathrm{cm}^{2}$ e tempo de 10 minutos. Foram produzidos três painéis por tratamento, totalizando 24 painéis. O plano experimental consistiu em quatro composições de painel e dois teores de sólidos da batida de cola, conforme a Tabela 1.

Após a prensagem, os painéis foram acondicionados na câmara climática à temperatura de $20+3^{\circ} \mathrm{C}$ e umidade relativa de $65+5 \%$. Finalizado o acondicionamento, foram retirados os corpos de prova 
Iwakiri et al. - Viabilidade de uso da madeira de Cupressus torulosa para

produção de painéis compensados

Tabela 1. Plano experimental.

Table 1. Experimental plan.

\begin{tabular}{lcc}
\hline Tratamento & Composição do painel / lâminas & Teor de sólidos (\%) \\
\hline T1: 100 C35 & $100 \%$ Cupressus torulosa & $35 \%$ \\
T2: 100 P35 & $100 \%$ Pinus taeda \\
T3: CPPPC35 & Capas: Cupressus torulosa + Miolo: Pinus taeda & \\
T4: PCCCP35 & Capas: Pinus taeda + Miolo: Cupressus torulosa & \\
T5: 100 C25 & $100 \%$ Cupressus torulosa \\
T6: 100 P25 & $100 \%$ Pinus taeda \\
T7: CPPPC25 & Capas: Cupressus torulosa + Miolo: Pinus taeda & \\
T8: PCCCP25 & Capas: Pinus taeda + Miolo: Cupressus torulosa & \\
\hline
\end{tabular}

para realização de ensaios de massa específica aparente, flexão estática (paralelo e perpendicular) e cisalhamento da linha de cola (teste seco, ciclo de fervura e fervura 72 horas), conforme as prescrições da norma EN 323, 310 e 314 (CEN, 1993a,b,c). Os ensaios de cisalhamento foram realizados na $1^{\text {a }}$ (capa) e $2^{\text {a }}$ (miolo) linha de cola, com a finalidade de avaliar as interfaces entre as lâminas de Cupressus e Pinus.

Os resultados foram submetidos à análise estatística por meio dos testes de kolmogorov-Smirnov e Bartlet, para verificação da normalidade e homogeneidade dos dados. Os efeitos da composição do painel e teor de sólidos da batida de cola foram avaliadas a partir da análise de variância em arranjo fatorial (4×2) e Teste de médias por Tukey ao nível de probabilidade de 95\%.

\section{RESULTADOS E DISCUSSÕES}

\section{Massa específica aparente dos painéis}

Na Tabela 2 estão apresentados os resultados médios da massa específica aparente dos painéis compensados obtidos para os diferentes tratamentos.

Os valores médios de massa específica aparente dos painéis variaram de $0,57 \mathrm{~g} / \mathrm{cm}^{3}$, para painéis produzidos com mistura de lâminas de Cupressus e Pinus, com $35 \%$ de sólidos a 0,61 g/ $\mathrm{cm}^{3}$, para painéis produzidos com todas as lâminas de Cupressus e 35\% de sólidos. Houve diferenças significativas entre os tratamentos, porém com pequenas variações entre os painéis.

Os valores de massa específica obtidos para compensados de Cupressus e de Pinus, foram superiores aos valores apresentados pela ABIMCI (2002) para compensados comerciais de Pinus de 0,49 a $0,56 \mathrm{~g} / \mathrm{cm}^{3}$.

Tabela 2. Resultados médios da massa específica aparente dos painéis.

Table 2. Average results of apparent density of plywood.

\begin{tabular}{|c|c|}
\hline Tratamento & Massa específica aparente $\left(\mathrm{g} / \mathrm{cm}^{3}\right)$ \\
\hline T1: 100C35 & $0,61 \mathrm{a}(6,20)$ \\
\hline T2: 100P35 & $0,59 \mathrm{ab}(6,34)$ \\
\hline T3: CPPPC35 & $0,57 \mathrm{~b}(6,08)$ \\
\hline T4: PCCCP35 & $0,57 \mathrm{~b}(5,84)$ \\
\hline T5: $100 \mathrm{C} 25$ & $0,59 \mathrm{ab}(4,62)$ \\
\hline T6: 100P25 & $0,60 \mathrm{ab}(4,48)$ \\
\hline T7: CPPPC25 & $0,60 \mathrm{ab}(4,91)$ \\
\hline T8: PCCCP25 & $0,60 \mathrm{ab}(5,03)$ \\
\hline
\end{tabular}

C: Cupressus torulosa; P: Pinus taeda; 35 e 25: teor de sólidos da batidade cola; médias seguidas de mesma letra na mesma coluna são estatisticamente iguais pelo Teste de Tukey a $95 \%$ de confiabilidade; valores entre parênteses indicam o coeficiente de variação.

Na Tabela 3 estão apresentados os resultados médios dos efeitos da composição do painel e do teor de sólidos da batida de cola sobre a massa específica aparente para os diferentes tratamentos.

Os resultados da análise fatorial indicaram que não há diferenças significativas entre as massas específicas para diferentes composições do painel e teor de sólidos da batida de cola. A composição do painel com todas as lâminas de Cupressus ou de Pinus, além da mistura destas espécies, não afetaram, significativamente, os resultados da massa específica dos painéis. Da mesma forma, o aumento no teor de sólidos da batida de cola, de $25 \%$ para 35\%, não resultaram em aumento da massa específica dos painéis. 
Tabela 3. Resultados médios do efeito da composição do painel e do teor de sólidos da batida de cola sobre a massa específica aparente.

Table 3. Average results of the effects of board composition and solid contents on the plywood density.

\begin{tabular}{ccc}
\hline \multicolumn{2}{c}{ Efeito principal } & \multicolumn{1}{c}{ Massa específica aparente $\left(\mathbf{g} / \mathbf{c m}^{3}\right)$} \\
\hline \multicolumn{3}{c}{ Composiçao do painel } \\
\hline $\begin{array}{c}100 \% \text { Cupressus torulosa } \\
\text { CPPPC }\end{array}$ & 0,60 a $(4,92)$ \\
PCCCP & 0,59 a $(7,16)$ \\
\hline & Teor de sólidos da batida de cola & 0,59 a $(5,13)$ \\
\hline $25 \%$ & 0,59 a $(4,61)$ \\
\hline $35 \%$ & 0,69 a $(6,43)$ \\
\hline
\end{tabular}

C: Cupressus torulosa; P: Pinus taeda; médias seguidas de mesma letra na mesma coluna são estatisticamente iguais pelo Teste de Tukey a $95 \%$ de confiabilidade; valores entre parênteses indicam o coeficiente de variação.

\section{Flexão estática paralela e perpendicular}

Na Tabela 4 estão apresentados os valores médios de módulo de ruptura e módulo de elasticidade em flexão estática, nas direções de ensaios paralelo e perpendicular dos painéis compensados.

Tabela 4. Resultados médios dos ensaios de flexão estática dos painéis compensados.

Table 4. Average results of static bending test of plywood.

\begin{tabular}{|c|c|c|c|c|}
\hline \multirow{2}{*}{ Tratamento } & \multicolumn{2}{|c|}{ Paralelo } & \multicolumn{2}{|c|}{ Perpendicular } \\
\hline & MOR (MPa) & MOE (MPa) & MOR (MPa) & MOE (MPa) \\
\hline T1: $100 \mathrm{C} 35$ & $\begin{array}{l}48,70 \mathrm{a} \\
(30,14)\end{array}$ & $\begin{array}{l}5.207 \mathrm{a} \\
(21,89)\end{array}$ & $\begin{array}{l}24,90 \mathrm{a} \\
(25,98)\end{array}$ & $\begin{array}{l}1.695 \mathrm{~b} \\
(15,64)\end{array}$ \\
\hline T2: 100P35 & $\begin{array}{c}46,06 \text { a } \\
(29,16)\end{array}$ & $\begin{array}{l}5.487 \mathrm{a} \\
(23,90)\end{array}$ & $\begin{array}{l}31,93 \text { a } \\
(26,39)\end{array}$ & $\begin{array}{c}2.251 \mathrm{ab} \\
(30,70)\end{array}$ \\
\hline T3: CPPPC35 & $\begin{array}{c}46,38 \text { a } \\
(30,91)\end{array}$ & $\begin{array}{l}4.952 \mathrm{a} \\
(21,95)\end{array}$ & $\begin{array}{l}27,45 \text { a } \\
(26,42)\end{array}$ & $\begin{array}{l}1.547 \mathrm{~b} \\
(32,96)\end{array}$ \\
\hline T4: РCCСР35 & $\begin{array}{l}45,70 \mathrm{a} \\
(32,30)\end{array}$ & $\begin{array}{l}4.683 \mathrm{a} \\
(22,08)\end{array}$ & $\begin{array}{l}28,24 \text { a } \\
(29,40)\end{array}$ & $\begin{array}{l}2,486 \text { a } \\
(30,43)\end{array}$ \\
\hline T5: 100C25 & $\begin{array}{l}43,04 \mathrm{a} \\
(32,02)\end{array}$ & $\begin{array}{l}4.861 \mathrm{a} \\
(23,69)\end{array}$ & $\begin{array}{l}30,37 \text { a } \\
(27,91)\end{array}$ & $\begin{array}{c}1.794 a b \\
(21,76)\end{array}$ \\
\hline T6: 100P25 & $\begin{array}{l}48,75 \mathrm{a} \\
(32,87)\end{array}$ & $\begin{array}{l}5.002 \mathrm{a} \\
(26,08)\end{array}$ & $\begin{array}{l}25,78 \text { a } \\
(28,81)\end{array}$ & $\begin{array}{c}2.015 \mathrm{ab} \\
(29,38)\end{array}$ \\
\hline T7: CPPPC25 & $\begin{array}{l}36,68 \text { a } \\
(28,99)\end{array}$ & $\begin{array}{l}5.054 \mathrm{a} \\
(22,96)\end{array}$ & $\begin{array}{l}27,92 \text { a } \\
(27,03)\end{array}$ & $\begin{array}{l}1.710 b \\
(23,31)\end{array}$ \\
\hline T8: РCCCP25 & $\begin{array}{l}53,36 \mathrm{a} \\
(28,91)\end{array}$ & $\begin{array}{l}5.187 \text { a } \\
(25,18)\end{array}$ & $\begin{array}{l}21,48 \mathrm{a} \\
(26,84)\end{array}$ & $\begin{array}{l}1.745 \mathrm{~b} \\
(24,45)\end{array}$ \\
\hline
\end{tabular}

C: Cupressus torulosa; P: Pinus taeda; 35 e 25: teor de sólidos da batida de cola; MOR: módulo de ruptura; MOE: módulo de elasticidade; médias seguidas de mesma letra na mesma coluna são estatisticamente iguais pelo Teste de Tukey a $95 \%$ de confiabilidade; valores entre parênteses indicam o coeficiente de variação.

Os valores médios de MOR e MOE paralelo dos painéis variaram, respectivamente, de 36,68 MPa (compensados de Cupressus na capa e Pinus no miolo com 25\% de sólidos) a 53,36 MPa (compensados de Pinus na capa e Cupressus no miolo com 25\% de sólidos); e, de $4.683 \mathrm{MPa}$ (compensados de Pinus na capa e Cupressus no miolo com 35\% de sólidos) a $5.487 \mathrm{MPa}$ (compensados de Pinus com 35\% de sólidos). Não foram constatadas diferenças significativas entre tratamentos, tanto para o MOR, quanto para o MOE paralelo.

Para o MOR e MOE perpendicular, os valores médios variaram, respectivamente, de 21,48 $\mathrm{MPa}$ (compensados de Pinus na capa e Cupressus no miolo com 25\% de sólidos) a 31,93 MPa (compensados de Pinus com 35\% de sólidos); e, de 1.547 MPa (compensados de Cupressus na capa e Pinus no miolo com 35\% de sólidos) a $2.486 \mathrm{MPa}$ (compensados de Pinus na capa e Cupressus no miolo com $35 \%$ de sólidos). Não foram constatadas diferenças significativas entre os tratamentos para o MOR perpendicular. Para o MOE perpendicular, os painéis produzidos com Pinus na capa e Cupressus no miolo, com 35\% de sólidos, apresentaram média estatisticamente igual em relação aos painéis produzidos com todas as lâminas de Pinus com 25 e 35\% de sólidos e, superior em relação aos demais tratamentos. 
Iwakiri et al. - Viabilidade de uso da madeira de Cupressus torulosa para

produção de painéis compensados

Os resultados obtidos nesta pesquisa estão compatíveis com as faixas de valores mencionados pela ABIMCI (2002) para painéis comerciais de Pinus, de 4.876 a 8.921 MPa (MOE paralelo); 23,4 a 52,7 MPa (MOR paralelo); 1.903 a $3.774 \mathrm{MPa}$ (MOE perpendicular); e 15,8 a 34,8 MPa (MOR perpendicular).

Os resultados obtidos para os compensados de Cupressus foram satisfatórios em comparação aos valores apresentados por alguns pesquisadores. Iwakiri et al. (2002) encontraram, para compensados de Pinus taeda, valores médios de MOE e MOR paralelo de 5.800 MPa e 58,40 MPa, respectivamente. Utilizando lâminas de Sequoia sempervirens para produção de compensados fenólicos, com diferentes gramaturas e teores de sólidos, Iwakiri et al. (2013) obtiveram valores médios de MOE e MOR paralelo na faixa de 3.188 a $3.651 \mathrm{MPa}$ e 24 a $29 \mathrm{MPa}$, respectivamente. Pinto e Iwakiri (2013) encontraram para compensados de Cryptomeria japônica, valores médios de MOE e MOR paralelo na faixa de 3.387 a $7.078 \mathrm{MPa}$ e 26 a $56 \mathrm{MPa}$, respectivamente.

Na Tabela 5 estão apresentados os resultados médios dos efeitos da composição do painel e do teor de sólidos da batida de cola sobre as propriedades de flexão estática.

Tabela 5. Resultados médios do efeito da composição do painel e do teor de sólidos da batida de cola sobre as propriedades de flexão estática.

Table 5. Average results of the effects of board composition and solid contents on the properties of static bending.

\begin{tabular}{|c|c|c|c|c|}
\hline Efeito principal & \multicolumn{2}{|c|}{ Paralelo } & \multicolumn{2}{|c|}{ Perpendicular } \\
\hline Composiçao do painel & MOR (MPa) & MOE (MPa) & MOR (MPa) & MOE (MPa) \\
\hline $100 \%$ Cupressus torulosa & $\begin{array}{l}45,87 \mathrm{a} \\
(32,45)\end{array}$ & $\begin{array}{l}5.034 \mathrm{a} \\
(19,70)\end{array}$ & $\begin{array}{l}27,64 \mathrm{a} \\
(26,90)\end{array}$ & $\begin{array}{c}1.745 \mathrm{ab} \\
(15,64)\end{array}$ \\
\hline $100 \%$ Pinus taeda & $\begin{array}{l}47,40 \mathrm{a} \\
(32,10)\end{array}$ & $\begin{array}{l}5.424 \text { a } \\
(29,64)\end{array}$ & $\begin{array}{l}28,85 \text { a } \\
(28,05)\end{array}$ & $\begin{array}{l}2.122 \mathrm{a} \\
(33,53)\end{array}$ \\
\hline CPPPC & $\begin{array}{l}41,53 \text { a } \\
(21,35)\end{array}$ & $\begin{array}{l}5.003 \text { a } \\
(23,46)\end{array}$ & $\begin{array}{l}27,69 \text { a } \\
(23,81)\end{array}$ & $\begin{array}{l}1.629 b \\
(27,01)\end{array}$ \\
\hline PCCCP & $\begin{array}{l}49,53 \mathrm{a} \\
(32,47)\end{array}$ & $\begin{array}{l}4.936 \mathrm{a} \\
(24,64)\end{array}$ & $\begin{array}{l}24,86 \text { a } \\
(30,24)\end{array}$ & $\begin{array}{l}2.116 \text { a } \\
(22,01)\end{array}$ \\
\hline \multicolumn{5}{|c|}{ Teor de sólidos da batida de cola } \\
\hline $25 \%$ & $\begin{array}{l}45,46 \text { a } \\
(31,10)\end{array}$ & $\begin{array}{l}5.026 \mathrm{a} \\
(25,33)\end{array}$ & $\begin{array}{l}26,39 \text { a } \\
(27,91)\end{array}$ & $\begin{array}{l}1.816 \mathrm{a} \\
(22,48)\end{array}$ \\
\hline $35 \%$ & $\begin{array}{l}46,71 \mathrm{a} \\
(30,81)\end{array}$ & $\begin{array}{l}5.172 \text { a } \\
(21,79)\end{array}$ & $\begin{array}{l}28,13 \mathrm{a} \\
(26,90)\end{array}$ & $\begin{array}{l}1.995 \mathrm{a} \\
(32,47)\end{array}$ \\
\hline
\end{tabular}

C: Cupressus torulosa; P: Pinus taeda; médias seguidas de mesma letra na mesma coluna são estatisticamente iguais pelo Teste de Tukey a $95 \%$ de confiabilidade; valores entre parênteses indicam o coeficiente de variação.

Os resultados da análise fatorial indicaram que não há diferenças significativas entre os valores médios de MOR e MOE paralelo e MOR perpendicular, para diferentes composições do painel. Para o MOE perpendicular, os painéis produzidos com lâminas de Pinus na capa e Cupressus no miolo apresentaram média estatisticamente igual, em relação aos painéis produzidos com todas as lâminas de Pinus e de Cupressus e, superior, em relação aos painéis produzidos com lâminas de Cupressus na capa e Pinus no miolo.

Quanto aos efeitos do teor de sólidos da batida de cola, não foram constatadas diferenças significativas entres os tratamentos.

\section{Cisalhamento da linha de cola}

Na Tabela 6 estão apresentados os valores médios de resistência da linha de cola ao cisalhamento nos ensaios a seco, ciclo de fervura e fervura 72 horas dos painéis compensados.

Os valores médios de tensões de cisalhamento nos ensaios a seco para a $1^{\text {a }}$ linha de cola (capa) e linha de cola mais interna (miolo), variaram, respectivamente, de 1,42 MPa (compensados de Pinus na capa e Cupressus no miolo com 25\% de sólidos) a 2,04 MPa (compensados de Cupressus na capa e Pinus no miolo com 35\% de sólidos); e, de 1,38 MPa (compensados de Pinus na capa e Cupressus no miolo com 35\% de sólidos) a 2,19 MPa (compensados de Pinus com 35\% de sólidos).

As percentagens de falhas na madeira variaram de $63 \%$ a $92 \%$, para a $1^{\text {a }}$ linha de cola, e de $70 \%$ a $96 \%$, para a $2^{\text {a }}$ linha de cola.

Todos os tratamentos apresentaram valores médios de resistência da linha de cola ao cisalhamento acima do valor mínimo de 1,0 MPa, conforme estabelecido pela norma EN 314-2 (1993). 
Tabela 6. Resultados médios dos ensaios de cisalhamento da linha de cola.

Table 6. Average results of glue line shear tests.

\begin{tabular}{|c|c|c|c|c|c|c|}
\hline \multirow[t]{2}{*}{$\mathrm{Tt}$} & \multicolumn{2}{|c|}{$\begin{array}{c}\text { Cisalhamento - seco } \\
(\mathrm{MPa})\end{array}$} & \multicolumn{2}{|c|}{$\begin{array}{c}\text { Cisalhamento - ciclo de fervura } \\
(\mathrm{MPa})\end{array}$} & \multicolumn{2}{|c|}{$\begin{array}{c}\text { Cisalhamento - fervura } 72 \mathrm{~h} \\
\text { (MPa) }\end{array}$} \\
\hline & Miolo & Capa & Miolo & Capa & Miolo & Capa \\
\hline $\mathrm{T} 1$ & $\begin{array}{l}1,58 b^{63} \\
(27,55)\end{array}$ & $\begin{array}{c}1,92 a^{81} \\
(37,15)\end{array}$ & $\begin{array}{c}0,78 a^{81} \\
(29,36)\end{array}$ & $\begin{array}{c}1,14 a b^{46} \\
(33,78)\end{array}$ & $\begin{array}{c}0,68 b c^{40} \\
(33,43)\end{array}$ & $\begin{array}{l}1,14 \mathrm{a}^{20} \\
(37,19)\end{array}$ \\
\hline T2 & $\begin{array}{c}1,77 a^{a}{ }^{91} \\
(28,24)\end{array}$ & $\begin{array}{c}2,19 a^{96} \\
(39,64)\end{array}$ & $\begin{array}{c}0,75 \text { abc }{ }^{11} \\
(31,19)\end{array}$ & $\begin{array}{c}0,74 b c^{50} \\
(40,75)\end{array}$ & $\begin{array}{c}0,82 a b^{17} \\
(38,16)\end{array}$ & $\begin{array}{c}0,59 c^{48} \\
(41,86)\end{array}$ \\
\hline T3 & $\begin{array}{c}2,04 \mathrm{a}^{86} \\
(27,73)\end{array}$ & $\begin{array}{c}1,86 a^{93} \\
(38,90)\end{array}$ & $\begin{array}{c}0,64 \mathrm{bc}^{7} \\
(30,63)\end{array}$ & $\begin{array}{c}0,80 b^{23} \\
(39,06)\end{array}$ & $\begin{array}{c}0,53 c^{16} \\
(35,66)\end{array}$ & $\begin{array}{c}0,76 a b c^{2} \\
(38,62)\end{array}$ \\
\hline $\mathrm{T} 4$ & $\begin{array}{c}1,49 b^{91} \\
(27,27)\end{array}$ & $\begin{array}{c}1,38 a^{82} \\
(27,46)\end{array}$ & $\begin{array}{c}0,82 a b^{41} \\
(27,46)\end{array}$ & $\begin{array}{c}1,26 a^{23} \\
(34,54)\end{array}$ & $\begin{array}{c}0,92 a^{41} \\
(32,78)\end{array}$ & $\begin{array}{c}0,98 a b c^{20} \\
(38,74)\end{array}$ \\
\hline T5 & $\begin{array}{l}1,46 b^{85} \\
(26,70)\end{array}$ & $\begin{array}{c}1,87 a^{84} \\
(33,16)\end{array}$ & $\begin{array}{c}0,89 a^{24} \\
(32,78)\end{array}$ & $\begin{array}{c}1,07 a^{26} \\
(41,72)\end{array}$ & $\begin{array}{c}0,73 \mathrm{abc}^{46} \\
(41,72)\end{array}$ & $\begin{array}{c}1,07 a b^{19} \\
(41,85)\end{array}$ \\
\hline T6 & $\begin{array}{c}1,78 a^{92}{ }^{92} \\
(28,61)\end{array}$ & $\begin{array}{c}1,57 a^{76} \\
(29,62)\end{array}$ & $\begin{array}{c}0,58 c^{17} \\
(31,29)\end{array}$ & $\begin{array}{c}0,59 c^{29} \\
(40,42)\end{array}$ & $\begin{array}{c}0,64 b c^{18} \\
(29,07)\end{array}$ & $\begin{array}{l}0,62 c^{16} \\
(44,56)\end{array}$ \\
\hline $\mathrm{T} 7$ & $\begin{array}{c}1,85 a^{2}{ }^{86} \\
(30,39)\end{array}$ & $\begin{array}{c}1,58 a^{70} \\
(29,98)\end{array}$ & $\begin{array}{c}0,74 \mathrm{abc}^{36} \\
(33,67)\end{array}$ & $\begin{array}{c}0,60 \mathrm{c}^{14} \\
(40,48\end{array}$ & $\begin{array}{c}0,74 \mathrm{abc}^{32} \\
(31,16)\end{array}$ & $\begin{array}{c}0,65 \text { bc }{ }^{19} \\
(40,25)\end{array}$ \\
\hline T8 & $\begin{array}{l}1,42 b^{84} \\
(28,31)\end{array}$ & $\begin{array}{c}1,89 a^{86} \\
(37,21)\end{array}$ & $\begin{array}{c}0,74 \mathrm{abc}^{18} \\
(32,25)\end{array}$ & $\begin{array}{c}0,83 \text { bc }{ }^{48} \\
(40,93)\end{array}$ & $\begin{array}{c}0,79 a b^{19} \\
(29,86)\end{array}$ & $\begin{array}{c}0,93 a b c c^{51} \\
(39,24)\end{array}$ \\
\hline
\end{tabular}

Tt: tratamentos, conforme plano experimental; médias seguidas de mesma letra na mesma coluna são estatisticamente iguais pelo Teste de Tukey a $95 \%$ de confiabilidade; valores entre parênteses indicam o coeficiente de variação; valores sobrescritos indicam a falha na madeira.

Para os ensaios em ciclo de fervura, os valores médios de tensões de cisalhamento para a $1^{\text {a }}$ linha de cola (capa) e linha de cola mais interna (miolo) variaram, respectivamente, de 0,58 MPa (compensados de Pinus com 25\% de sólidos) a 0,89 MPa (compensados de Cupressus com 25\% de sólidos); e, de 0,59 MPa (compensados de Pinus com 25\% de sólidos) a 1,26 MPa (compensados de Pinus na capa e Cupressus no miolo com 35\% de sólidos).

As percentagens de falhas na madeira variaram de $7 \%$ a $81 \%$ para a $1^{\text {a }}$ linha de cola, e de $14 \%$ a $46 \%$ para a $2^{\text {a }}$ linha de cola.

Na avaliação comparativa com os requisitos normativos da EN 314-2 (1993), apenas os painéis produzidos com todas as lâminas de Cupressus, e com lâminas de Pinus na capa e Cupressus no miolo, ambos com 35\% de sólidos, atenderam ao requisito mínimo para tensões de cisalhamento de 0,6 a 1,0 MPa com percentagem mínimo de $40 \%$ de falhas na madeira.

Para os ensaios em ciclo de fervura 72 horas, os valores médios de tensões de cisalhamento para a $1^{\text {a }}$ linha de cola (capa) e linha de cola mais interna (miolo) variaram, respectivamente, de 0,53 MPa (compensados de Cupressus na capa e Pinus no miolo com 35\% de sólidos) a 0,92 MPa (compensados de Pinus na capa e Cupressus no miolo com 35\% de sólidos); e, de 0,59 MPa (compensados de Pinus com 35\% de sólidos) a 1,14 MPa (compensados de Cupressus com 35\% de sólidos).

As percentagens de falhas na madeira variaram de $16 \%$ a $46 \%$ para a $1^{\text {a }}$ linha de cola, e de $2 \%$ a $51 \%$ para a $2^{\text {a }}$ linha de cola.

Os ensaios realizados após pré-tratamentos em água quente (ciclo de fervura e fervura $72 \mathrm{~h}$ ) apresentaram menores percentagens de falhas na madeira em comparação aos ensaios a seco, indicando o efeito da água e temperatura na degradação da linha de cola.

$\mathrm{Na}$ avaliação comparativa com os requisitos normativos da EN 314-2 (1993), apenas os painéis produzidos com todas as lâminas de Cupressus, com 35\% e 25\% de sólidos, e com lâminas de Pinus na capa e Cupressus no miolo com 35\% de sólidos, atenderam ao requisito mínimo para tensões de cisalhamento de 0,6 a 1,0 MPa, com percentagem mínimo de $40 \%$ de falhas na madeira.

Com algumas exceções, os resultados de cisalhamento da $1^{\text {a }}$ linha de cola (capa) indicaram que não houve diferenças significativas entre as interfaces da colagem de lâminas de Cupressus e de Pinus, demonstrando a possibilidade de mistura destas espécies para produção de compensados.

De uma maneira geral, os resultados obtidos neste estudo foram satisfatórios em comparação aos dados apresentados na literatura para algumas espécies provenientes de plantios florestais. Pinto e Iwakiri (2013) encontraram, para compensados fenólicos de Cryptomeria japônica, valores de tensões de cisalhamento para ensaios a seco na faixa de 1,02 a 1,20 MPa, e após ciclo de fervura de 0,70 a 0,80 MPa; Iwakiri et al. (2013) encontraram para compensados de Sequoia sempervirens, valores médios na faixa de 1,13 a 1,37 MPa para ensaios a seco, e de 0,66 a 0,94 MPa após ciclo de fervura. 
Importantes constatações podem ser feitas no que se refere às tensões de cisalhamento obtidas para a $1^{\text {a }}$ (capa) e $2^{\text {a }}$ (miolo) linha de cola. Com algumas exceções, os valores de tensões de cisalhamento da linha de cola mais externa foram superiores aos obtidos para a linha de cola mais interna, indicando os efeitos da maior rapidez na transferência de calor e da migração de vapor da linha de cola mais próxima dos pratos quentes da prensa.

Na Tabela 7 estão apresentados os resultados médios dos efeitos da composição do painel e do teor de sólidos da batida de cola na resistência da linha de cola ao cisalhamento.

Tabela 7. Resultados médios do efeito da composição do painel e do teor de sólidos da batida de cola sobre a resistência da linha de cola ao cisalhamento.

Table 7. Average results of the effects of board composition and solid contents on the glue line shear strength.

\begin{tabular}{|c|c|c|c|c|c|c|}
\hline \multirow[t]{2}{*}{$\mathrm{Tt}$} & \multicolumn{2}{|c|}{$\begin{array}{c}\text { Cisalhamento - seco } \\
\text { (MPa) }\end{array}$} & \multicolumn{2}{|c|}{$\begin{array}{l}\text { Cisalhamento - ciclo de fervura } \\
(\mathrm{MPa})\end{array}$} & \multicolumn{2}{|c|}{$\begin{array}{c}\text { Cisalhamento - fervura } 72 \mathrm{~h} \\
(\mathrm{MPa})\end{array}$} \\
\hline & Miolo & Capa & Miolo & Capa & Miolo & Capa \\
\hline $\mathrm{C}$ & $\begin{array}{c}1,52 \text { bc }{ }^{74} \\
(18,70)\end{array}$ & $\begin{array}{c}1,89 a^{83} \\
(34,20)\end{array}$ & $\begin{array}{c}0,84 a^{27} \\
(29,23)\end{array}$ & $\begin{array}{c}1,10 a^{36} \\
(28,54)\end{array}$ & $\begin{array}{c}0,70 b^{43} \\
(29,65)\end{array}$ & $\begin{array}{l}1,11 \mathrm{a}^{19} \\
(29,44)\end{array}$ \\
\hline$P$ & $\begin{array}{c}1,78 a^{91} \\
(28,97)\end{array}$ & $\begin{array}{l}1,81 a^{86} \\
(31,85)\end{array}$ & $\begin{array}{c}0,66 b^{14} \\
(29,92)\end{array}$ & $\begin{array}{c}0,66 b^{39} \\
(41,89)\end{array}$ & $\begin{array}{c}0,73 a b^{18} \\
(33,75)\end{array}$ & $\begin{array}{l}0,61 \mathrm{c}^{32} \\
(47,15)\end{array}$ \\
\hline CPC & $\begin{array}{l}1,94 a^{86} \\
(28,72)\end{array}$ & $\begin{array}{l}1,72 a^{85} \\
(33,32)\end{array}$ & $\begin{array}{c}0,69 b^{21} \\
(36,04)\end{array}$ & $\begin{array}{c}0,70 b^{26} \\
(43,75)\end{array}$ & $\begin{array}{l}0,64 c^{24} \\
(37,92)\end{array}$ & $\begin{array}{l}0,71 \mathrm{bc}^{9} \\
(39,20)\end{array}$ \\
\hline PCP & $\begin{array}{l}1,46 c^{88} \\
(21,34)\end{array}$ & $\begin{array}{l}1,63 a^{89} \\
(46,19)\end{array}$ & $\begin{array}{c}0,78 \mathrm{ab}^{30} \\
(24,88)\end{array}$ & $\begin{array}{l}1,05 a^{36} \\
(30,01)\end{array}$ & $\begin{array}{l}0,85 a^{30} \\
(24,03)\end{array}$ & $\begin{array}{c}0,95 \mathrm{ab}^{36} \\
(32,39)\end{array}$ \\
\hline \multicolumn{7}{|c|}{ Teor de sólidos da batida de cola } \\
\hline $25 \%$ & $\begin{array}{l}1,63 a^{87} \\
(28,98)\end{array}$ & $\begin{array}{l}1,72 a^{79} \\
(30,24)\end{array}$ & $\begin{array}{l}0,74 a^{24} \\
(33,46)\end{array}$ & $\begin{array}{c}0,77 b^{29} \\
(41,64)\end{array}$ & $\begin{array}{l}0,72 a^{29} \\
(28,82)\end{array}$ & $\begin{array}{l}0,82 a^{26} \\
(40,25)\end{array}$ \\
\hline $35 \%$ & $\begin{array}{l}1,72 a^{83} \\
(27,47)\end{array}$ & $\begin{array}{l}1,84 a^{91} \\
(39,87)\end{array}$ & $\begin{array}{l}0,75 a^{28} \\
(28,91)\end{array}$ & $\begin{array}{c}0,99 a^{36} \\
(35,31)\end{array}$ & $\begin{array}{l}0,74 a^{28} \\
(35,81)\end{array}$ & $\begin{array}{l}0,87 a^{23} \\
(38,55)\end{array}$ \\
\hline
\end{tabular}

C: Cupressus torulosa; P: Pinus taeda; CPC: capas de Cupressus e miolo de Pinus; PCP: capas de Pinus e miolo de Cupressus; Médias seguidas de mesma letra na mesma coluna são estatisticamente iguais pelo Teste de Tukey a 95\% de confiabilidade; Valores entre parênteses indicam o coeficiente de variação; Valores sobrescritos indicam a falha na madeira.

Os resultados da análise fatorial sobre os efeitos da composição do painel sobre a resistência da linha de cola indicaram que houve diferenças significativas entre as médias para todos os pré-tratamentos realizados.

Nos ensaios de cisalhamento da primeira linha de cola após pré-tratamento a seco, os painéis produzidos com lâminas de Cupressus na capa e Pinus no miolo apresentaram média estatisticamente igual em relação aos painéis produzidos com todas as lâminas de Pinus e, superior, às demais composições. Já na linha de cola mais interna, não foram constatadas diferenças significativas entre os tratamentos.

Nos ensaios de cisalhamento da primeira linha de cola, após ciclo de fervura, os painéis produzidos com todas as lâminas de Cupressus apresentaram média estatisticamente igual em relação aos painéis produzidos com lâminas de Cupressus na capa e Pinus no miolo e, superior, às demais composições. Já na linha de cola mais interna, os painéis produzidos com todas as lâminas de Cupressus, e lâminas de Pinus na capa e Cupressus no miolo, apresentaram médias estatisticamente iguais entre si, e superiores às demais composições.

Nos ensaios de cisalhamento da primeira linha de cola após fervura 72h, os painéis produzidos com lâminas de Pinus na capa e Cupressus no miolo apresentaram média estatisticamente igual em relação aos painéis produzidos com todas as lâminas de Pinus e, superior, às demais composições. Já na linha de cola mais interna, os painéis produzidos com lâminas de Pinus na capa e Cupressus no miolo, apresentaram média estatisticamente igual em relação aos painéis com todas as lâminas de Cupressus e, superior, às demais composições.

Quanto aos efeitos do teor de sólidos da batida de cola, não foram constatadas diferenças significativas entres os tratamentos, com exceção dos ensaios de cisalhamento da $1^{\text {a }}$ linha de cola após ciclo de fervura. Neste pré-tratamento, os painéis produzidos com 35\% de sólidos apresentaram média estatisticamente superior em relação aos painéis com $25 \%$ de sólidos. 


\section{CONCLUSÕES}

Os painéis compensados produzidos com lâminas de Cupressus torulosa apresentaram massa específica superior aos painéis comerciais de Pinus.

Os resultados de cisalhamento da linha de cola não evidenciaram diferenças significativas entre as composições de painéis com lâminas de Cupressus e de Pinus.

O aumento no teor de sólidos da batida de cola de 25\% para 35\% não influenciaram nos resultados de cisalhamento da linha de cola e flexão estática dos painéis, indicando a possibilidade de menor consumo de adesivo na produção de compensados.

As avaliações gerais dos resultados obtidos permitem concluir que Cupressus torulosa apresenta grande potencial para produção de painéis compensados, puro, ou em mistura com Pinus taeda.

\section{REFERÊNCIAS}

ABIMCI - ASSOCIAÇÃO BRASILEIRA DA INDÚSTRIA DE MADEIRA PROCESSADA MECANICAMENTE. Painéis de compensado de pinus - Catálogo Técnico. Curitiba: ABIMCI, 2007. 16 p.

BALDWIN, R. F. Plywood and veneer based products: manufacturing practices. San Francisco: Miller Freeman, $1995.388 \mathrm{p}$.

BENDSTEN, B. A. Properties of wood from improved and intensively managed trees. In: ECONOMICS \& FINANCIAL MANAGEMENT AND TIMBER PRODUCTION TECHNICAL COMITEES OFTHE FOREST PRODUCTS RESEARCH SOCIETY. Atlanta, 1978. Proceedings... Atlanta: 1978. 78 p.

BORTOLETTO JÚNIOR, G.; BELINI, U. L. Produção de lâminas e manufatura de compensados a partir da madeira de guapuruvu (Schizolobium parahyba Blake) proveniente de um plantio misto de espécies nativas. Cerne, Lavras, v.8, n. 2, p. 1-16, 2002.

CEN - EUROPEAN COMITTEE FOR STANDARDIZATION. European Satandard EM 310 - Determination of modulus of elasticity and modulus of rupture in static bending. Bruxels, 1993a.

CEN - EUROPEAN COMITTEE FOR STANDARDIZATION. European Satandard EM 314-1 - Determination of shear bonding strength. Bruxels, 1993b.

CEN - EUROPEAN COMITTEE FOR STANDARDIZATION. European Satandard EM 323 - Determination of density. Bruxels, 1993c.

HASLETT. A. Properties and utilization of exotic specialty timbers grown in New Zealand. Part III: Cypress. Forest Research Institute Bulletin, v.119, p. 1-12, 1986.

IWAKIRI, S.; CUNHA, A. B.; TRIANOSKI, R.; BRAZ, R. L.; CASTRO, V. G.; KAZMIERCZAK, S.; PINHEIRO, E.; RANCATTI, H.; SANCHES, F. L. Produção de painéis compensados fenólicos com lâminas de madeira de Sequoia sempervirens. Floresta e Ambiente, Seropédica, v.20, n.2, p.264-270, 2013.

IWAKIRI, S.; DEL MENEZZI, C. H. S.; LAROCA, C.; VENSON, I.; MATOSKI, S. S. Produção de compensados de Pinus taeda e Pinus caribaea com resina fenol-formaldeído. Cerne, Lavras, v.11, n.2, p.092-097, 2002.

MARRA, A. A. Technology of wood bonding: principles in practice. New York: Van Nostrand Reinhold, 1992. $453 \mathrm{p}$.

OKINO, E. Y. A.; SOUZA, M. R.; SANTANA, M. A. E.; ALVES, M. V. S.; SOUSA, M. E.; TEIXEIRA, D. E. Physicomechanical properties and decay resistance of cupressus spp. Cement-bonded particleboards. Cement and Concrete Composites, v. 27, p. 333-338, 2005.

PINTO, J. A.; IWAKIRI, S. Estudo sobre a viabilidade de uso da madeira de Cryptomeria japônica para produção de painéis compensados. Scientia Forestalis, Piracicaba, v. 41, n. 97, p.29-37, 2013.

SELLERS, T. Plywood and adhesive technology. New York: Marcel Dekker, 1993. 661 p.

TRIANOSKI, R.; IWAKIRI, S.; MATOS, J. L. M.; HIGA, A. R.; BRAZ, R. K. Avaliação das propriedades de painéis compensados de Melia azedarach L. produzidos com diferentes gramaturas e tempos de prensagem. Árvore, Viçosa, v. 39, n. 4, p. 759-768, 2015.

Recebido em: 25/10/2017

Aceito em: 17/05/2018

Sci. For., Piracicaba, v. 46, n. 120, p. 638-645, dez. 2018

DOI: dx.doi.org/10.18671/scifor.v46n120.12 